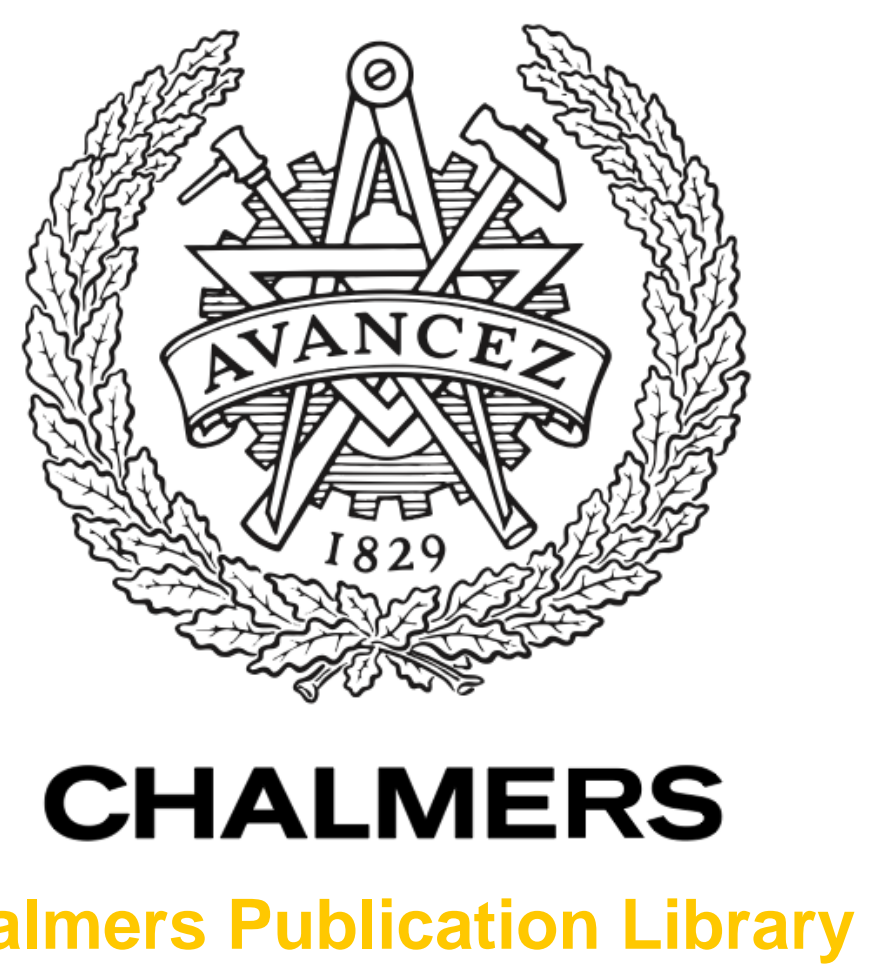

Braided Convolutional Codes: A Class of Spatially Coupled Turbo-Like Codes

This document has been downloaded from Chalmers Publication Library (CPL). It is the author's version of a work that was accepted for publication in:

International Conference on Signal Processing and Communications (SPCOM)

Citation for the published paper:

Lentmaier, M. ; Moloudi, S. ; Graell i Amat, A. (2014) "Braided Convolutional Codes: A

Class of Spatially Coupled Turbo-Like Codes". International Conference on Signal

Processing and Communications (SPCOM)

Downloaded from: http://publications.lib.chalmers.se/publication/200795

Notice: Changes introduced as a result of publishing processes such as copy-editing and formatting may not be reflected in this document. For a definitive version of this work, please refer to the published source. Please note that access to the published version might require a subscription. 


\title{
Braided Convolutional Codes - A Class of Spatially Coupled Turbo-Like Codes
}

\author{
Michael Lentmaier ${ }^{\dagger}$, Saeedeh Moloudi ${ }^{\dagger}$, and Alexandre Graell i Amat $^{\ddagger}$ \\ $\dagger$ Department of Electrical and Information Technology, Lund University, Lund, Sweden \\ $\ddagger$ Department of Signals and Systems, Chalmers University of Technology, Gothenburg, Sweden \\ \{saeedeh.moloudi,michael.lentmaier\}@eit.lth.se, alexandre.graell@chalmers.se
}

\begin{abstract}
In this paper, we investigate the impact of spatial coupling on the thresholds of turbo-like codes. Parallel concatenated and serially concatenated convolutional codes as well as braided convolutional codes (BCCs) are compared by means of an exact density evolution (DE) analysis for the binary erasure channel (BEC). We propose two extensions of the original BCC ensemble to improve its threshold and demonstrate that their BP thresholds approach the maximum-a-posteriori (MAP) threshold of the uncoupled ensemble. A comparison of the different ensembles shows that parallel concatenated ensembles can be outperformed by both serially concatenated and BCC ensembles, although they have the best BP thresholds in the uncoupled case.
\end{abstract}

\section{INTRODUCTION}

It is well known that spatially coupled LDPC codes exhibit a threshold saturation phenomenon: the threshold of an iterative belief propagation (BP) decoder, obtained by density evolution (DE), is improved to that of the optimal maximum-a-posteriori (MAP) decoder [1], [2]. As a consequence, it is possible to achieve capacity with simple regular LDPC codes, which show without spatial coupling a significant gap between the BP and the MAP threshold.

The concept of spatial coupling is not limited to LDPC codes. Recently it has been shown that spatial coupling has a similar effect on the thresholds of turbo-like codes, i.e., concatenated convolutional codes that can be described by sparse graphical models. Some block-wise spatially coupled ensembles of parallel concatenated codes (SC-PCCs) and serially concatenated codes (SC-SCCs) were introduced in [3]. For the binary erasure channel (BEC) it is possible to derive exact $\mathrm{DE}$ equations for these ensembles from the transfer functions of the component decoders [4], [5] and perform a threshold analysis, analogously to [1], [2]. The numerical results in [3] suggest that threshold saturation occurs if the coupling memory is chosen sufficiently large. A similar threshold analysis has been performed in [6] for braided convolutional codes (BCCs) [7], another class of turbo-like codes which have an inherent spatially coupled structure.

The aim of this paper is to give an overview of spatially coupled turbo-like codes and compare the thresholds of SCPCCs, SC-SCCs and BCCs. We first present the ensembles considered in [3] and [6]. Then we generalize the original BCC ensemble (type-I BCC) to larger coupling memories

This work was supported in part by the Swedish Research Council (VR) under grant \#621-2013-5477. and demonstrate that threshold saturation occurs. Furthermore, inspired by the SC-PCC construction in [3], we introduce and analyze a new BCC ensemble (type-II BCC) in which not only the parity symbols but also the information symbols are coupled over several time instants. The thresholds are further improved by this construction, i.e., the gap to the MAP threshold is smaller for a given coupling memory and vanishes as the memory is increased.

\section{Spatially Coupled Turbo-Like Codes}

In this section, we describe how the concept of spatial coupling can be applied to turbo-like codes. We consider the parallel and serially concatenated convolutional codes introduced in [3] as well as the braided convolutional codes from [7] and [6]. We assume that at each time instant $t$ an information sequence $\boldsymbol{u}_{t}$ is encoded into a code sequence $\boldsymbol{v}_{t}$, which is transmitted over the channel. The fundamental idea of spatial coupling is that these transmitted sequences are interconnected in the encoding process instead of being processed independently. In order to achieve such an interconnection, some information symbols and/or code symbols from previous time instants $t^{\prime}<t$ serve as inputs of the component encoders at time $t$. The coupling memory $m$ defines the range of $t^{\prime}$, i.e., $t^{\prime} \in\{t-m, \ldots, t\}$. In what follows we assume that the encoding starts at $t=1$ and is terminated at $t=L$ in such a way that $\boldsymbol{v}_{L+1}=\mathbf{0}$. The value $L$ is called the coupling length. Analogously to conventional convolutional codes, this leads to a rate loss that becomes smaller as $L$ increases.

\section{A. Parallel Concatenated Convolutional Codes}

Fig. 1 shows the factor graph of two parallel concatenated rate- $1 / 2$ convolutional encoders with coupling memory $m=1$. In order to enable a coupled structure the information sequence $\boldsymbol{u}_{t}$ is split randomly into two sequences, $\boldsymbol{u}_{t, \mathrm{~A}}$ and $\boldsymbol{u}_{t, \mathrm{~B}}$. At time $t$ the information sequences $\left(\boldsymbol{u}_{t, \mathrm{~A}}, \boldsymbol{u}_{t-1, \mathrm{~B}}\right)$ and $\left(\boldsymbol{u}_{t, \mathrm{~B}}, \boldsymbol{u}_{t-1, \mathrm{~A}}\right)$ are used by the upper encoder $C_{\mathrm{U}}$ and lower encoder $C_{\mathrm{L}}$ to produce the parity sequences $\boldsymbol{v}_{t}^{\mathrm{U}}$ and $\boldsymbol{v}_{t}^{\mathrm{L}}$, respectively. The transmitted code sequence is equal to $\boldsymbol{v}_{t}=\left(\boldsymbol{u}_{t}, \boldsymbol{v}_{t}^{\mathrm{U}}, \boldsymbol{v}_{t}^{\mathrm{L}}\right)$. For more details about this code ensemble, including a generalization to larger coupling memories, we refer the reader to [3].

\section{B. Serial Concatenated Convolutional Codes}

The factor graph of two serially concatenated rate-1/2 convolutional encoders with coupling memory $m=1$ is shown 


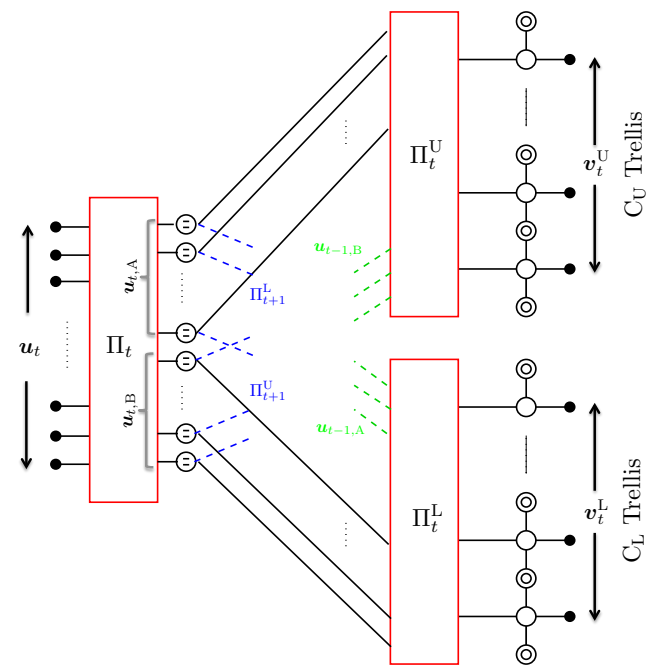

Fig. 1. Parallel concatenation: factor graph at time $t$.

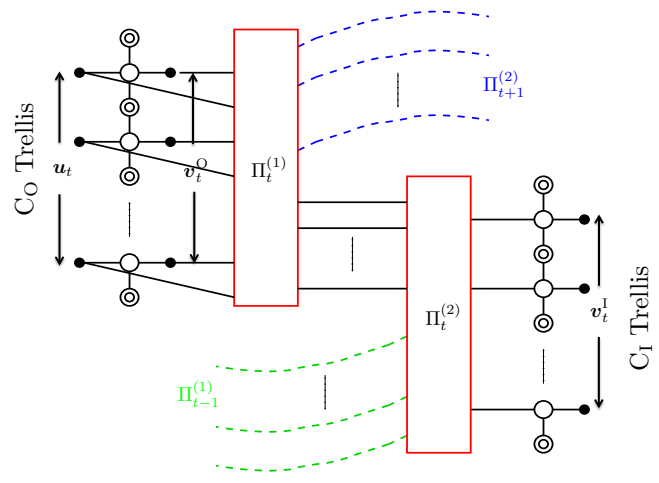

Fig. 2. Serial concatenation: factor graph at time $t$.

in Fig. 2. In this case the code sequence $\boldsymbol{v}_{t}^{\mathrm{O}}$ of the outer encoder $C_{\mathrm{O}}$ is randomly divided into two parts, $\tilde{\boldsymbol{v}}_{t, \mathrm{~A}}^{\mathrm{O}}$ and $\tilde{\boldsymbol{v}}_{t, \mathrm{~B}}^{\mathrm{O}}$. The input of the inner encoder $C_{\mathrm{I}}$ at time $t$ is $\left(\tilde{\boldsymbol{v}}_{t, \mathrm{~A}}^{\mathrm{O}}, \tilde{\boldsymbol{v}}_{t-1, \mathrm{~B}}^{\mathrm{O}}\right)$. The transmitted code sequence $\boldsymbol{v}_{t}$ is equal to the output of the inner encoder, $\boldsymbol{v}_{t}^{\mathrm{I}}$. For more details about this code ensemble, including a generalization to larger coupling memories, we refer to [3].

\section{Braided Convolutional Codes (Type-I)}

Similar to turbo codes, the encoder of a BCC is divided into two component encoders $C_{\mathrm{U}}$ and $C_{\mathrm{L}}$, with the special feature that the parity sequence of one component encoder is used as input of the other component encoder.

Fig. 3 shows the factor graph of a BCC with coupling memory $m=1$, consisting of two systematic rate- $2 / 3$ convolutional encoders, denoted by $C_{\mathrm{U}}$ and $C_{\mathrm{L}}$. Their parity sequences are given by $\boldsymbol{v}_{t}^{\mathrm{U}}$ and $\boldsymbol{v}_{t}^{\mathrm{L}}$, respectively. The first input of the upper encoder $C_{\mathrm{U}}$ at time $t$ is the information sequence $\boldsymbol{u}_{t}$. The second input is the previously generated parity sequence $\boldsymbol{v}_{t-1}^{\mathrm{L}}$ of $C_{\mathrm{L}}$, after being reordered by a permutation $\Pi_{t}^{\mathrm{U}}$. Likewise, the two inputs of the lower encoder $C_{\mathrm{L}}$ are $\boldsymbol{u}_{t}$ and $\boldsymbol{v}_{t-1}^{\mathrm{U}}$, which are both properly reordered by the permutations $\Pi_{t}$ and $\Pi_{t}^{\mathrm{L}}$, respectively. The transmitted code sequence is

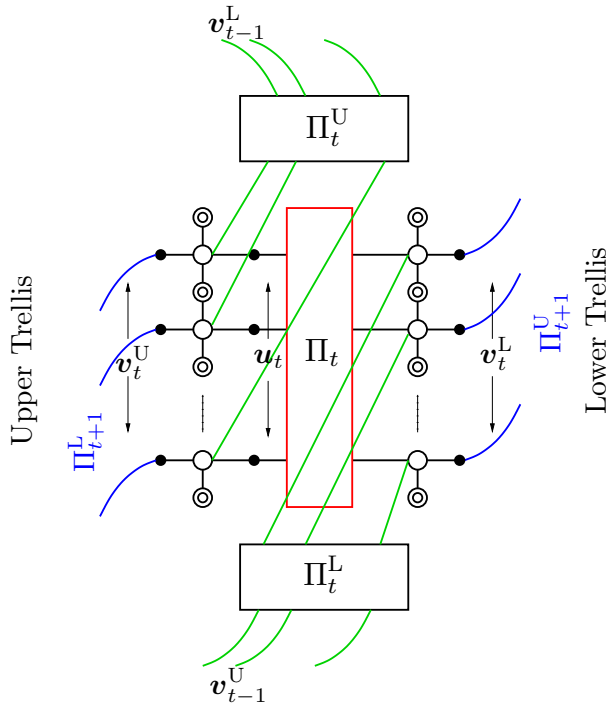

Fig. 3. Braided convolutional codes (Type-I): factor graph at time $t$.

$\boldsymbol{v}_{t}=\left(\boldsymbol{u}_{t}, \boldsymbol{v}_{t}^{\mathrm{U}}, \boldsymbol{v}_{t}^{\mathrm{L}}\right)$. An encoder block diagram of the resulting BCC with overall rate $R=1 / 3$ is illustrated in Fig. 4(a).

The above described ensemble is equivalent to the original block-wise BCC ensemble introduced in [7] and analyzed in [6]. Throughout this paper we will refer to these codes as type-I BCCs.

\section{TyPe-I BRAided CONVOLUTIONAL CODES: Generalization to Larger Coupling Memories}

In this section, we generalize the type-I BCCs described in Section II-C to coupling memory $m>1$.

Fig. 5 shows the encoder of type-I BCCs with coupling memory $m=2 \kappa-1$. At time $t$, the coded sequence of $C_{\mathrm{U}}$, $\boldsymbol{v}_{t}^{\mathrm{U}}$, is randomly divided into $m$ parts by the use of permutation $\Pi_{t}^{(\mathrm{U}, \mathrm{p})}$ followed by a demultiplexer. These $m$ sequences are shown in the right side of Fig. 5 and denoted by $\boldsymbol{v}_{t, j}^{\mathrm{U}}, j=$ $1, \ldots, 2 \kappa-1$. At time $t$, the sequences $\boldsymbol{v}_{t-j, j}^{\mathrm{U}}$ are multiplexed and reordered by the permutation $\Pi_{t}^{\mathrm{L}}$ to create a new sequence, which is used as a second input of $C_{\mathrm{L}}$. Likewise, the coded sequence of the lower encoder at time $t$ is split into $m$ parts, $\boldsymbol{v}_{t, j}^{\mathrm{L}}, j=1, \ldots, m$. The sequences $\boldsymbol{v}_{t-j, j}^{\mathrm{L}}$ are then merged and reordered by permutation $\Pi_{t}^{\mathrm{U}}$ to create the second input of $C_{\mathrm{U}}$. On the other hand, for type-I BCCs, the first input of $C_{\mathrm{U}}$ and $C_{\mathrm{L}}$ are the information bits and reordered information bits, respectively.

\section{A. Density Evolution Analysis}

The extrinsic erasure probability of the $k$ th output of $C_{\mathrm{U}}$ is denoted by $p_{\mathrm{U}, \mathrm{k}}, k=1,2,3$. These erasure probabilities $p_{\mathrm{U}, \mathrm{k}}$, at iteration $i$ and time $t$, can be written as

$$
\begin{aligned}
& p_{\mathrm{U}, 1}^{(i, t)}=f_{\mathrm{U}, 1}\left(q_{\mathrm{L}, 1}^{(i-1)}, q_{\mathrm{L}, 2}^{(i-1)}, q_{\mathrm{L}, 3}^{(i-1)}\right), \\
& p_{\mathrm{U}, 2}^{(i, t)}=f_{\mathrm{U}, 2}\left(q_{\mathrm{L}, 1}^{(i-1)}, q_{\mathrm{L}, 2}^{(i-1)}, q_{\mathrm{L}, 3}^{(i-1)}\right), \\
& p_{\mathrm{U}, 3}^{(i, t)}=f_{\mathrm{U}, 3}\left(q_{\mathrm{L}, 1}^{(i-1)}, q_{\mathrm{L}, 2}^{(i-1)}, q_{\mathrm{L}, 3}^{(i-1)}\right) .
\end{aligned}
$$




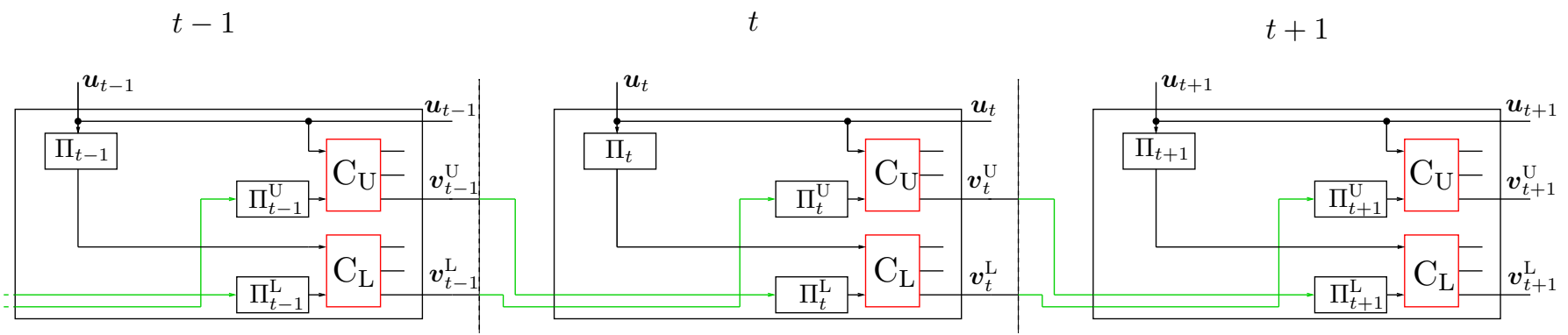

(a)

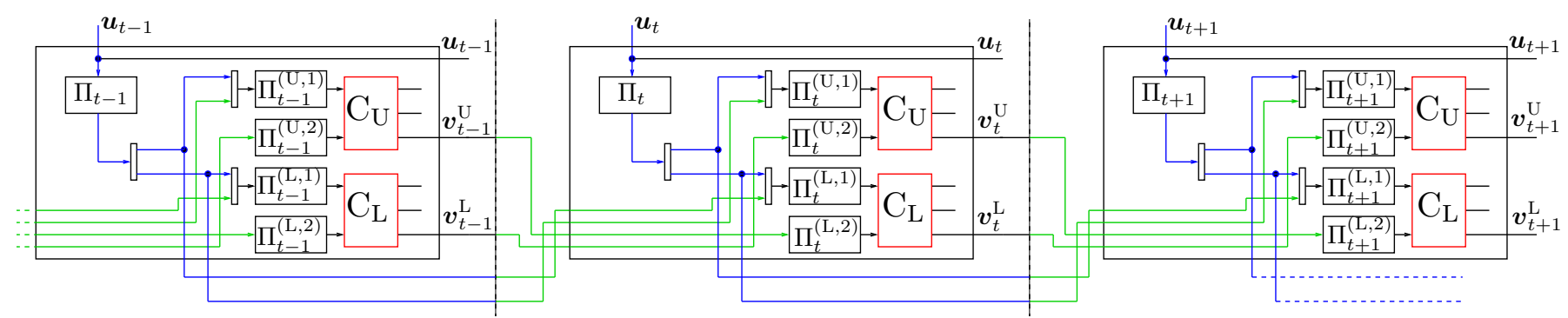

(b)

Fig. 4. Block diagram of the braided convolutional encoder with coupling memory $m=1$. (a) Type-I BCCs (b) Type-II BCCs.

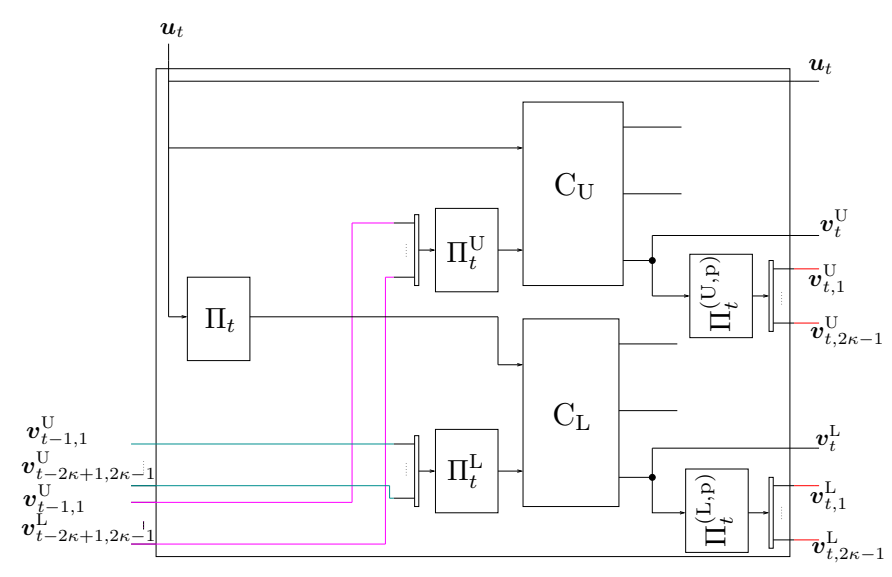

Fig. 5. Block diagram of the encoder of type-I BCCs with $m=2 \kappa-1$.

where $f_{\mathrm{U}, \mathrm{k}}$ is the decoder transfer function of $C_{\mathrm{U}}$ for the $k$ th output of the decoder, and $q_{\mathrm{L}, \mathrm{k}}$ are the input erasure probabilities. In [6] we described the method to derive the exact expression for the transfer functions $f_{\mathrm{U}, \mathrm{k}}$ and compute DE equations for the case $m=1$. Here, we generalize the equations to coupling memory $m$.

The input erasure probabilities $q_{\mathrm{L}, 1}^{(i-1)}, q_{\mathrm{L}, 2}^{(i-1)}$ and $q_{\mathrm{L}, 3}^{(i-1)}$ in (1), (2) and (3) are as follow,

$$
\begin{gathered}
q_{\mathrm{L}, 1}^{(i-1)}=\epsilon \cdot p_{\mathrm{L}, 1}^{(i-1, t)}, \\
q_{\mathrm{L}, 2}^{(i-1)}=\epsilon \cdot \frac{\sum_{j=1}^{m} p_{\mathrm{L}, 3}^{(i-1, t-j)}}{m}, \\
q_{\mathrm{L}, 3}^{(i-1)}=\epsilon \cdot \frac{\sum_{j=1}^{m} p_{\mathrm{L}, 2}^{(i-1, t+j)}}{m},
\end{gathered}
$$

where $p_{\mathrm{L}, \mathrm{k}}^{(i, t)}$ is the extrinsic erasure probability of the $k$ th output of $C_{\mathrm{L}}$ at iteration $i$ and time $t$. Note that $p_{\mathrm{L}, \mathrm{k}}^{(i, t)}$ is equal to zero for $t<0$ and $t>L$ and it is 1 for $i=0$. The equations for the lower decoder are obtained by simply interchanging the indices $\mathrm{U}$ and $\mathrm{L}$ with each other in (1)-(6)

Finally, the a-posteriori erasure probability on information bits at iteration $i$ and time $t$ is

$$
p_{\mathrm{a}}^{(i, t)}=\epsilon \cdot p_{\mathrm{U}, 1}^{(i, t)} \cdot p_{\mathrm{L}, 1}^{(i, t)} .
$$

\section{Type-II Braided Convolutional Codes: COUPLING OF INFORMATION SYMBOLS}

For simplicity, we first describe the coupling with coupling memory $m=1$ and we then consider the generalization to $m>1$.

\section{A. Coupling of Information Symbols}

The factor graph of type-II BCCs with $m=1$ is shown in Fig. 6. Consider a chain of $L$ encoders between time instants $t=1$ and $t=L$. According to Fig. 6, divide randomly the information sequence $\boldsymbol{u}_{t}$ at time $t$ into two parts, $\boldsymbol{u}_{t, \mathrm{~A}}$ and $\boldsymbol{u}_{t, \mathrm{~B}}$. The information sequence $\left(\boldsymbol{u}_{t, \mathrm{~A}}, \boldsymbol{u}_{t-1, \mathrm{~B}}\right)$, properly reordered by a permutation $\Pi_{t}^{(\mathrm{U}, 1)}$ is then used as the first input of encoder $C_{\mathrm{U}}$. Likewise, the sequence at the first input of encoder $C_{\mathrm{L}}$ is $\left(\boldsymbol{u}_{t, \mathrm{~B}}, \boldsymbol{u}_{t-1, \mathrm{~A}}\right)$, properly reordered by the permutation $\Pi_{t}^{(\mathrm{L}, 1)}$. The second inputs of $C_{\mathrm{U}}$ and $C_{\mathrm{L}}$ are the same as that of the original braided codes, $\boldsymbol{v}_{t-1}^{\mathrm{L}}$ and $\boldsymbol{v}_{t-1}^{\mathrm{U}}$, reordered by permutations $\Pi_{t}^{(\mathrm{U}, 2)}$ and $\Pi_{t}^{(\mathrm{L}, 2)}$, respectively. This coupling method is also illustrated in Fig. 4(b). In the figure, blue lines represent bits from the current time instant and green lines represent bits from the previous time instant. 


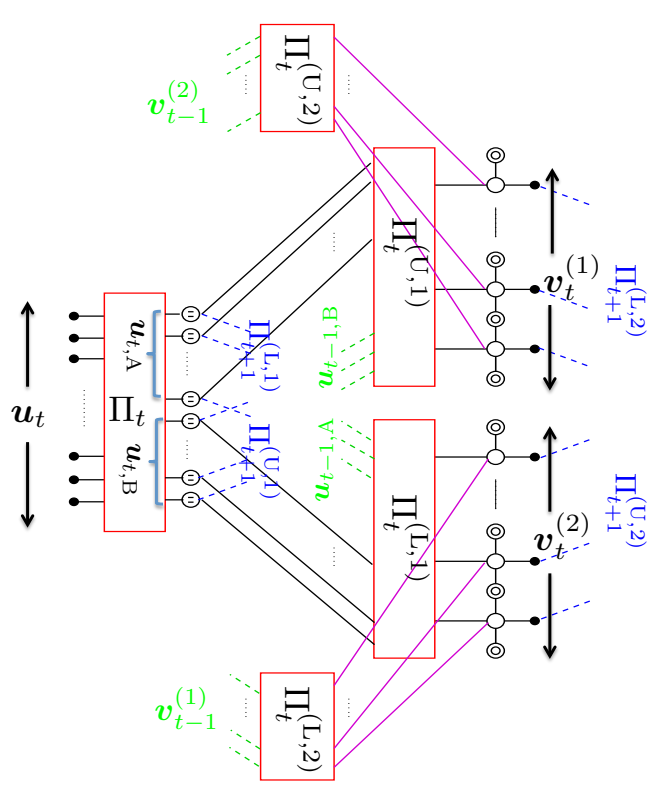

Fig. 6. Braided convolutional codes (Type-II): factor graph at time $t$.

\section{B. Generalization to Larger Coupling Memories}

For simplicity, we limit ourselves to odd values of $m, m=$ $2 \kappa-1$ for some $\kappa \geq 1$.

Fig. 7 shows the general form of type-II BCCs. In the general case, the information sequences $\boldsymbol{u}_{t}, \boldsymbol{u}_{t-1}, \ldots, \boldsymbol{u}_{t-2 \kappa+1}$ and parity sequences $\boldsymbol{v}_{t-1}^{\mathrm{L}}, \boldsymbol{v}_{t-2}^{\mathrm{L}}, \ldots, \boldsymbol{v}_{t-2 \kappa+1}^{\mathrm{L}}$ from different time instants contribute to the inputs of $C_{\mathrm{U}}$ at time $t$. Likewise, sequences $\boldsymbol{u}_{t}, \boldsymbol{u}_{t-1}, \ldots, \boldsymbol{u}_{t-2 \kappa+1}$ and $\boldsymbol{v}_{t-1}^{\mathrm{U}}, \boldsymbol{v}_{t-2}^{\mathrm{U}}, \ldots, \boldsymbol{v}_{t-2 \kappa+1}^{\mathrm{U}}$ contribute to the inputs of $C_{\mathrm{L}}$. This is achieved by randomly dividing the information sequence $\boldsymbol{u}_{t}$ and the parity sequences of $C_{\mathrm{U}}$ and $C_{\mathrm{L}}, \boldsymbol{v}_{t}^{\mathrm{U}}$ and $\boldsymbol{v}_{t}^{\mathrm{L}}$, into the sequences $\boldsymbol{u}_{t, j}, j=1, \ldots, 2 \kappa$, and $\boldsymbol{v}_{t, l}^{\mathrm{U}}, \boldsymbol{v}_{t, l}^{\mathrm{L}}, l=1, \ldots, 2 \kappa-1$, with permutations $\Pi_{t}, \Pi_{t}^{(\mathrm{U}, \mathrm{p})}$ and $\Pi_{t}^{(L, p)}$, respectively. The first input of $C_{\mathrm{U}}$ at time $t$ is the sequence obtained by multiplexing and reordering the sequences $\boldsymbol{u}_{t-j+1, j}$ by the permutation $\Pi_{t}^{(\mathrm{U}, 1)}$. The second input of $C_{\mathrm{U}}$ is the sequence obtained by multiplexing and reordering (through permutation $\Pi_{t}^{(\mathrm{U}, 2)}$ ) the sequences $\boldsymbol{v}_{t-l, l}^{\mathrm{L}}$.

The lower encoder $C_{\mathrm{L}}$ receives as first input the complementary set of information sequences $\boldsymbol{u}_{t-j+1, j^{\prime}}, j=$ $1, \ldots, 2 \kappa, j^{\prime}=(\kappa+j-1 \bmod 2 \kappa)+1$ in a symmetric fashion, multiplexed and reordered by $\Pi_{t}^{(\mathrm{L}, 1)}$. The second input of $C_{\mathrm{L}}$ is the sequence obtained by multiplexing and reordering (through $\Pi_{t}^{(\mathrm{U}, 2)}$ ) the sequences $\boldsymbol{v}_{t-l, l}^{\mathrm{U}}$. It follows that the information sequence $\boldsymbol{u}_{t, j}$ is used by $C_{\mathrm{U}}$ at time $t+j-1$ and by $C_{\mathrm{L}}$ at time $t+(\kappa+j-1 \bmod 2 \kappa)$. The encoder in Fig. 6 and Fig. 4(b) corresponds to the special case $\kappa=1$.

\section{Density Evolution Analysis}

Considering type-II BCCs with $m=1$, the input erasure probabilities at the input of the upper decoder (to be used in (1), (2) and (3)) are

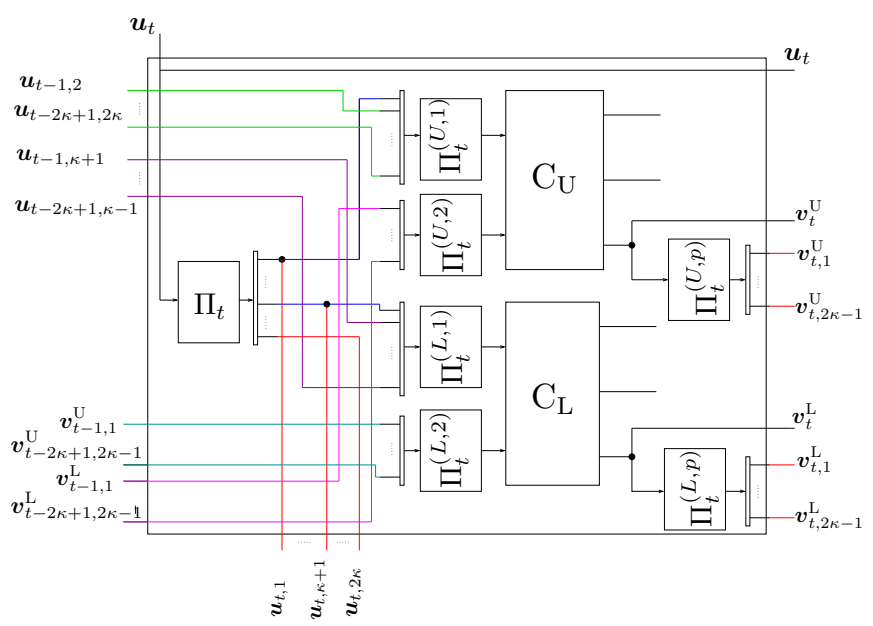

Fig. 7. Block diagram of the encoder of type-II BCCs with $m=2 \kappa-1$.

$$
\begin{gathered}
q_{\mathrm{L}, 1}^{(i-1)}=\epsilon \cdot \frac{p_{\mathrm{L}, 1}^{(i-1, t-1)}+p_{\mathrm{L}, 1}^{(i-1, t+1)}}{2}, \\
q_{\mathrm{L}, 2}^{(i-1)}=\epsilon \cdot p_{\mathrm{L}, 3}^{(i-1, t-1)}, \\
q_{\mathrm{L}, 3}^{(i-1)}=\epsilon \cdot p_{\mathrm{L}, 2}^{(i-1, t+1)} .
\end{gathered}
$$

We remark that these equations are identical to the ones in [6] for type-I BCCs except for (8).

Finally, the a-posteriori erasure probability on information bits at time $t$ and iteration $i$ is

$$
p_{\mathrm{a}}^{(i, t)}=\epsilon \cdot \frac{p_{\mathrm{U}, 1}^{(i, t)} p_{\mathrm{L}, 1}^{(i, t+1)}+p_{\mathrm{U}, 1}^{(i, t+1)} p_{\mathrm{L}, 1}^{(i, t)}}{2} .
$$

It is possible to generalize (8)-(11) to $m=2 \kappa-1$. We obtain

$$
q_{\mathrm{L}, 1}^{(i-1)}=\epsilon \cdot \frac{p_{\mathrm{L}, 1}^{(i-1, t-\kappa)}+p_{\mathrm{L}, 1}^{(i-1, t+\kappa)}}{2},
$$

while $q_{\mathrm{L}, 2}^{(i-1)}$ and $q_{\mathrm{L}, 3}^{(i-1)}$ are the same as (5) and (6) for type-I BCCs (see Section III-A) by setting $m=2 \kappa-1$.

For the lower decoder we can obtain similar expressions by interchanging the indices $\mathrm{U}$ and $\mathrm{L}$ in (8)-(10) and (12).

The a posteriori erasure probability on the information bits at time $t$ and iteration $i$ (7) becomes

$$
p_{\mathrm{a}}^{(i, t)}=\epsilon \cdot \frac{\sum_{j=1}^{2 \kappa} p_{\mathrm{U}, 1}^{(i, t+j-1)} p_{\mathrm{L}, 1}^{(i, t+(\kappa+j-1) \bmod 2 \kappa)}}{2 \kappa} .
$$

\section{RESUlts AND Discussion}

In this section, we present the BP thresholds for the two BCC ensembles considered in Section III and IV and compare them to SC-PCCs and SC-SCCs [3]. In particular, we consider BCCs with the two identical 4-state rate-2/3 component encoders used in [7], with generator matrix

$$
\boldsymbol{G}(D)=\left(\begin{array}{ccc}
1 & 0 & \frac{1}{1+D+D^{2}} \\
0 & 1 & \frac{1+D^{2}}{1+D+D^{2}}
\end{array}\right)=\left(\begin{array}{ccc}
1 & 0 & 1 / 7 \\
0 & 1 & 5 / 7
\end{array}\right)
$$


TABLE I

THRESHOLDS FOR BCCS $(R=1 / 3)$

\begin{tabular}{lcccccc}
\hline & \multirow{2}{*}{$\epsilon_{\mathrm{BP}}$} & $\epsilon_{\mathrm{MAP}}$ & \multicolumn{4}{c}{$\epsilon_{\mathrm{SC}}$} \\
& & & $m=1$ & $m=3$ & $m=5$ & $m=7$ \\
\hline Type-I & 0.55414 & 0.66539 & 0.66094 & 0.66447 & 0.66506 & 0.66524 \\
Type-II & 0.55414 & 0.66539 & 0.66534 & 0.66538 & 0.66539 & 0.66539 \\
\hline
\end{tabular}

TABLE II

THRESHOLDS FOR SC-PCCS AND PUNCTURED SC-SCCs $(R=1 / 3)$

\begin{tabular}{lccccc}
\hline & $\epsilon_{\mathrm{BP}}$ & $\epsilon_{\mathrm{MAP}}$ & \multicolumn{3}{c}{$\epsilon_{\mathrm{SC}}$} \\
& & & $m=1$ & $m=3$ & $m=5$ \\
\hline SC-PCCs & 0.64282 & 0.65538 & 0.65538 & 0.65538 & 0.65538 \\
SC-SCCs & 0.61184 & 0.66154 & 0.65190 & 0.66140 & 0.66153 \\
\hline
\end{tabular}

Likewise, for SC-PCCs and SC-SCCs we consider two identical 4-state rate-1/2 component encoders with generator matrix $\boldsymbol{G}=(1,5 / 7)$ (in octal notation). In order to compare the SC-SCCs with the other ensembles we increase their rate to $R=1 / 3$ using the random puncturing procedure described in [3]. All presented thresholds correspond to the stationary case $L \rightarrow \infty$, which lower bounds the thresholds for finite $L$. For small $L$ the threshold can be considerably larger but at the expense of a higher rate loss.

In Table I we give the BP threshold $\epsilon_{\mathrm{SC}}$ for BCCs of type-I and type-II with different coupling memory $m$. We also report in the table the BP threshold $\epsilon_{\mathrm{BP}}$ and the MAP threshold $\epsilon_{\mathrm{MAP}}$ of the uncoupled ensembles. The MAP threshold was computed applying the area theorem [8]. Note that the value for type-I with $m=1$ corresponds to the original ensemble considered in [6]. It can be observed that $\epsilon_{\mathrm{SC}}$ can be improved by increasing $m$ and it is expected that threshold saturation occurs. We can also see that the thresholds are better for typeII BCCs and are very close to the MAP threshold already for small coupling memory $m$.

The corresponding thresholds for SC-PCCs and punctured SC-SCCs are presented in Table II. Note that the puncturing rates are taken from [3] and are not necessarily the best possible choices. An optimization of these coefficients could lead to improvements regarding either the BP or the MAP threshold. We can observe that SC-PCCs have the best BP threshold in the uncoupled case but have a MAP threshold that is relatively poor. With spatial coupling a saturation to this MAP threshold occurs already for small coupling memory $m$. BCCs, on the other hand, have the worst BP threshold without coupling but a better MAP threshold than both SC-PCCs and SC-SCCs. In terms of the thresholds $\epsilon_{\mathrm{SC}}$ with spatial coupling we can see that BCCs of type-I and type-II outperform the other ensembles for all coupling memories. This confirms that BCCs are a powerful class of codes. Note that asymptotically they are also known to have superior distance properties (their minimum distance grows linearly with the block length [7]).

\section{CONCLUSiOnS}

In this paper we investigated the impact of spatial coupling on the BP decoding threshold of turbo-like codes. BCCs are a powerful class of turbo-like codes which are closely related to LDPC codes and product codes. We introduced two novel BCC ensembles (type-I and type-II) that generalize the original ensembles in [7]. For these ensembles we derived exact density evolution recursions for the BEC, which allowed us to numerically evaluate the thresholds for different coupling memories $m$ and compare them to the thresholds of parallel and serially concatenated convolutional codes. For all three classes of codes it can be observed that the BP threshold $\epsilon_{\mathrm{SC}}$ improves with increasing $m$, and we assume that a saturation to the MAP threshold $\epsilon_{\mathrm{MAP}}$ occurs. The best thresholds are obtained for the novel BCC ensemble of type-II. Interestingly, the gap between the BP threshold $\epsilon_{\mathrm{BP}}$ and the MAP threshold $\epsilon_{\mathrm{MAP}}$ is largest for the BCCs, which perform worst in the uncoupled case.

The considered examples of turbo-like codes demonstrate that the concept of spatial coupling opens some new degrees of freedom in the design of codes on graphs: instead of optimizing the component encoder characteristics for BP decoding it is possible to optimize the MAP decoding threshold and rely on the threshold saturation effect of spatial coupling. Powerful code ensembles with strong distance properties can then perform close to capacity with low-complexity iterative decoding.

\section{REFERENCES}

[1] S. Kudekar, T.J. Richardson, and R.L. Urbanke, "Threshold saturation via spatial coupling: Why convolutional LDPC ensembles perform so well over the BEC,' IEEE Trans. Inf. Theory, vol. 57, no. 2, pp. $803-834$, Feb. 2011.

[2] M. Lentmaier, A. Sridharan, D.J. Costello, Jr., and K.Sh. Zigangirov, "Iterative decoding threshold analysis for LDPC convolutional codes," IEEE Trans. Inf. Theory, vol. 56, no. 10, pp. 5274-5289, Oct. 2010.

[3] S. Moloudi, M. Lentmaier, and A. Graell i Amat, "Spatially coupled turbo codes," submitted to 8th International Symposium on Turbo Codes \& Iterative Information Processing, 2014.

[4] B.M. Kurkoski, P.H. Siegel, and J.K. Wolf, "Exact probability of erasure and a decoding algorithm for convolutional codes on the binary erasure channel," in Proc. IEEE Global Telecommunications Conference, 2003. GLOBECOM '03., Dec. 2003, vol. 3.

[5] J. Shi and S. ten Brink, "Exact EXIT functions for convolutional codes over the binary erasure channel," in Proceedings of the 44th Allerton Conference on Communication, Control, and Computing, Monticello, IL, USA, 2006.

[6] S. Moloudi and M. Lentmaier, "Density evolution analysis of braided convolutional codes on the erasure channel," in Proc. IEEE International Symposium on Information Theory, Honolulu, HI, USA, July 2014.

[7] W. Zhang, M. Lentmaier, K.Sh. Zigangirov, and D.J. Costello, Jr., "Braided convolutional codes: a new class of turbo-like codes," IEEE Trans. Inf. Theory, vol. 56, no. 1, pp. 316-331, Jan. 2010.

[8] C. Measson, A. Montanari, T.J. Richardson, and R. Urbanke, "The generalized area theorem and some of its consequences," IEEE Trans. Inf. Theory, vol. 55, no. 11, pp. 4793-4821, Nov. 2009. 